\title{
Is the Binary Search Faster when Two Variables are Added in the Middle of the Data?
}

Djasen Tjendry, Wirawan Istiono

Universitas Multimedia Nusantara

Indonesia

\{djasen@student.umn.ac.id\} \{wirawan.istiono@umn.ac.id\}

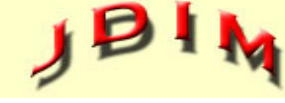

Journal of Digital Information Management
ABSTRACT: Modern architecture has developed new algorithms for large and distributed data, In this digital era, algorithms which intended to quickly process, search, and sort data are very much needed by IT developers. This research will discuss the modification of Binary Search Algorithm, which is previously known for its existing low, mid, and high variables. This research will discuss how we can modify the binary search algorithm by adding two variables- the variable mid-low and mid-high. It will show how by adding two variables between the mid variable could improve the performance of the Binary Search Algorithm.

Subject Categories and Descriptors

[B.2.4 High-Speed Arithmetic]: Algorithms; [H.3.3 Information Search and Retrieval]

General Terms: Binary Variables, Binary Search, Data Processing

Keywords: Data Search, Binary Search, Algorithm, Modified Algorithm

Received: 29 September 2019, Revised 22 December 2019, Accepted 3 January 2020

Review Metrics: Review Scale- 0/6, Review Score- 4.65, Interreviewer consistency: $76 \%$

DOI: $10.6025 / \mathrm{jdim} / 2020 / 18 / 2 / 57-64$

\section{Introduction}

Technology in this digital era has been developed rapidly, insisting human to adjust themselves to the challenges of this era. The technology development is growing rapidly so that almost of every human activity is associated with digital technology, the development of programming algorithms is increasing rapidly to enable human to use and develop the technology nowadays (Marszalek, 2017). Many algorithms were developed for processing data such as processing, searching, and sorting algorithms.

This paper will mainly discuss the algorithms to search for data. The searching process is a process that is usually done in daily activities - usually performed to look for something with the only aim to determine whether the data exists in the data set or not, sometimes we may want the sought data position (B, 2016; Pushpa \& Vinod, 2007; Van Sandt, Chronis, \& Patel, 2019). There are many algorithms to find the data, for example, well-known as a sequential search, interpolation search, exponential search, and binary search. This research will discuss the Binary Search algorithm that is well known in the programming field to search for data. This algorithm is relatively efficient, making it one of the most widely used methods to solve a variety of development problems. Binary search only works on a data set that has been sorted. In other words, to be able to use the binary search, the data must be sorted first. 
The Binary Search algorithm is well-known for being efficient and fast, but in this study, this algorithm will be modified, which was previously known to have the variables low, mid, and high (Mehlhorn \& Sanders, 2008), which serves as a variable that helps to find the data that is needed to be searched for, with the results being the value that is located in the middle position. And if it's not in the middle position, repetition will be done until the low variable and the high variable is in the same position if it happens, it means the searched value is not present in the database or the array (Rahim, Nurarif, Ramadhan, Aisyah, \& Purba, 2017; Schwarz, Maurice, Gruss, \& Mangard, 2017).

Based on the curiosity and the desire while studying the data processing algorithm such as searching and sorting algorithms, the desire to learn more about binary search algorithm and how to modify this basic search algorithm to make the searching is faster. By looking at the pattern of the workings of binary search that uses three variables which are increasingly conical to find the answer (Pooja, 2015), as well as inspiration when learning parallel binary search algorithm that uses a parallel system that executes odd and even indexes that divide the data into several parts (Mullins, Gennifer, Farrell, \& Baldwin, 2017), so that we get inspiration on how to find the desired data through the available array indexes, so we do experiment with using additional two variables between the mid variable are mid-low, and mid-high so that algorithm can divide the index in the array into four parts so that modified binary search can search by coning faster.

This research target with a modified binary search algorithm, that will have five variables namely low, mid-low, mid, mid-high, high variables, is expected to improve the performance of the binary algorithm and the searching process of the data, compared using only three variables. This research aims to prove that with adding two new variables between mid-variables will speed up the process of a data search..

\section{Method}

\subsection{Prepare Data to be used}

Now, data with enough numbers will be needed to be able to clearly see the comparison of the speed of the modification of the binary search algorithm, and even with other searching algorithms, then, an array containing 100,000 numeric data that are already sorted will be used because this method requires data that will be used to already be sorted (Lin, 2019; P. Parmar \& Kumbharana, 2015; Tremblay \& Sorenson, 1984). This paper will generate data by using the JavaScript programming language because it is relatively easier to understand. Then by looping and printing as many as 100,000 data, which will generate 100.000 numeric data that is already sorted, which will be used to test the binary searching algorithm that this research will discuss. When 100.000 -sorted data is prepared, it will be now ready for usage for this research.

\section{Binary Search Algorithm Modifications}

This research will use the binary search algorithm that originally only used the low, mid, and high variables as the basic algorithm for the development of modified binary search. The method used in this paper to measure the duration of the algorithm process runs from the beginning to get the results will be sought by the use of JavaScript function performance.now (), which can measure the duration of these algorithms during testing in this research.

This research will discuss how to modify the binary search algorithm by simply adding two additional variables, which are mid-low and mid-high between the mid variable to speed up the searching process of a binary search algorithm, using the results of making the data previously entered into a variable array. This research will use JavaScript programming language to show how to create a binary search algorithm modification, as shown below.

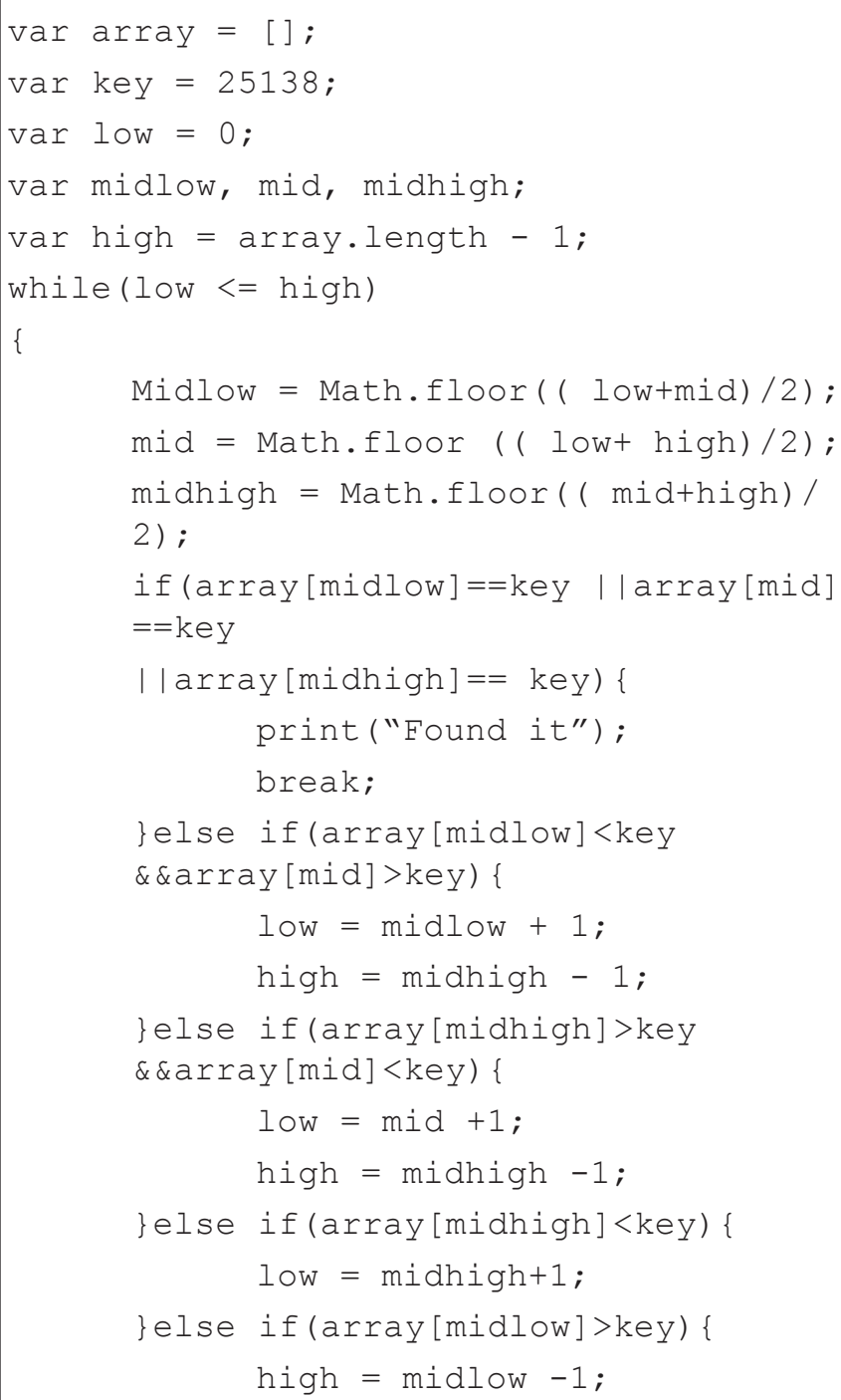

The sample code above will search the number 25138 from 100.000 sorted numerical data, and then it provides 


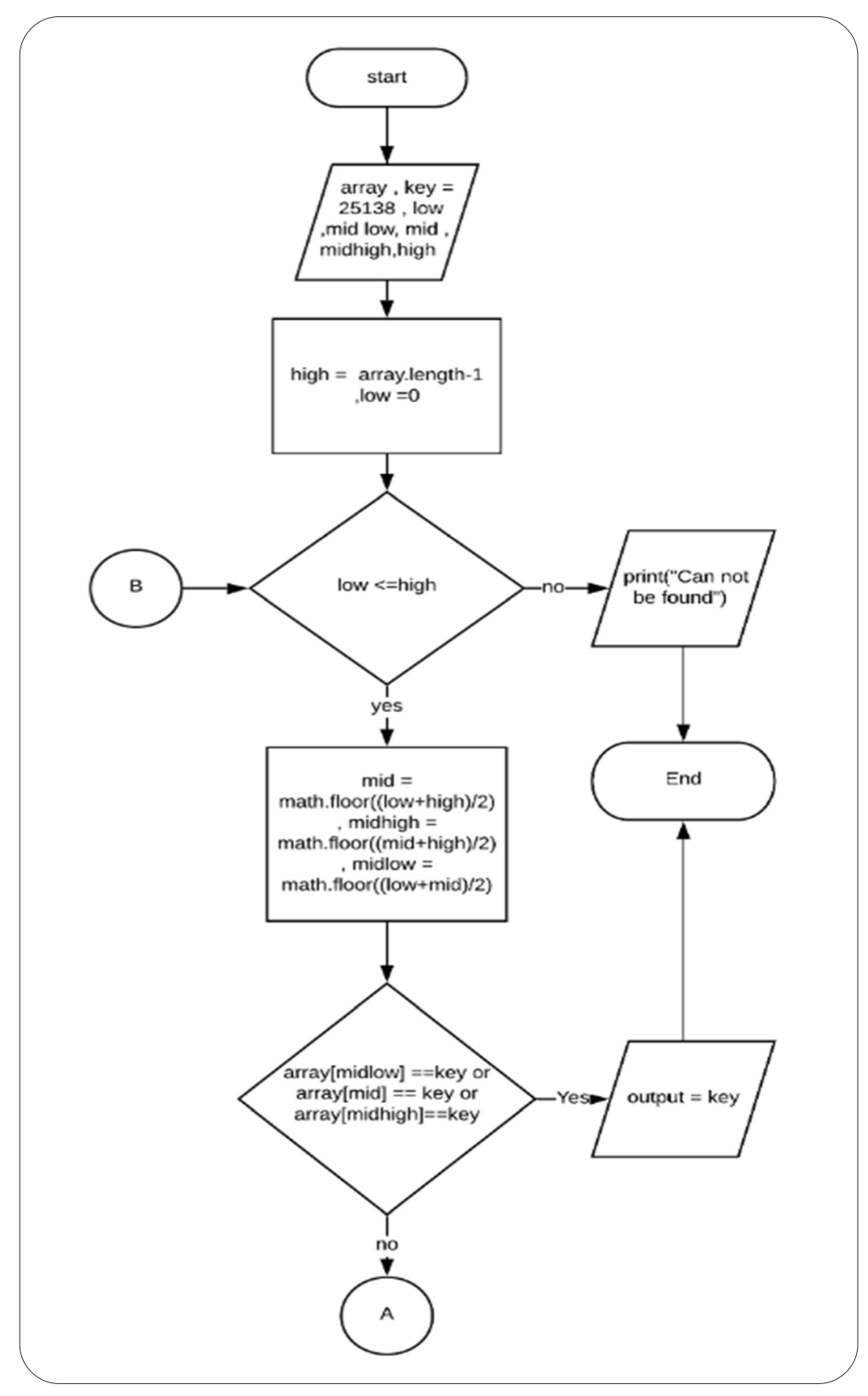

Figure 1. First Part Flowchart of modified binary search

three main variables; mid, mid-low, and mid-high and after that will do looping until the low value is bigger than the high variable, as long as the low variable is less than high variable. In addition, the next section will show the flowchart of binary search algorithm modification, to make it easier to understand the flow of the data search process this algorithm modification.

Figure 1 shows the first part flowchart modified binary search algorithm, wherein this first part does initialize variable for preparing calculation and initialize high and low value, where high level got from array length, and the low variable will always zero. After that, will do a comparison between low and high variable, as long as the low variable is less than high variable then will go calculation to search mid, mid-high and mid-low variable from low and high calculation variable. Where low get from low plus high variable and divide two, and mid-high got from mid plus high variable divide two, and mid-low got from low plus mid variable and divided two, this step is the main step because we add two new mid variable, and after that will do checking all three variable mid; mid, mid-low and mid-high variable, if one of variable mid is matching with search key so that output will show the result.

The flowcharts are shown in Figure 2 are modified binary search, which is connected by connectors $A$ and $B$, which have the same logic as the pseudocode previously described.

The addition of the variables of mid-low and mid-high is expected to accelerate the narrowing of the binary search process, utilizing a variable mid-low and mid-high is between mid-variables, as for some of the following circumstances: 


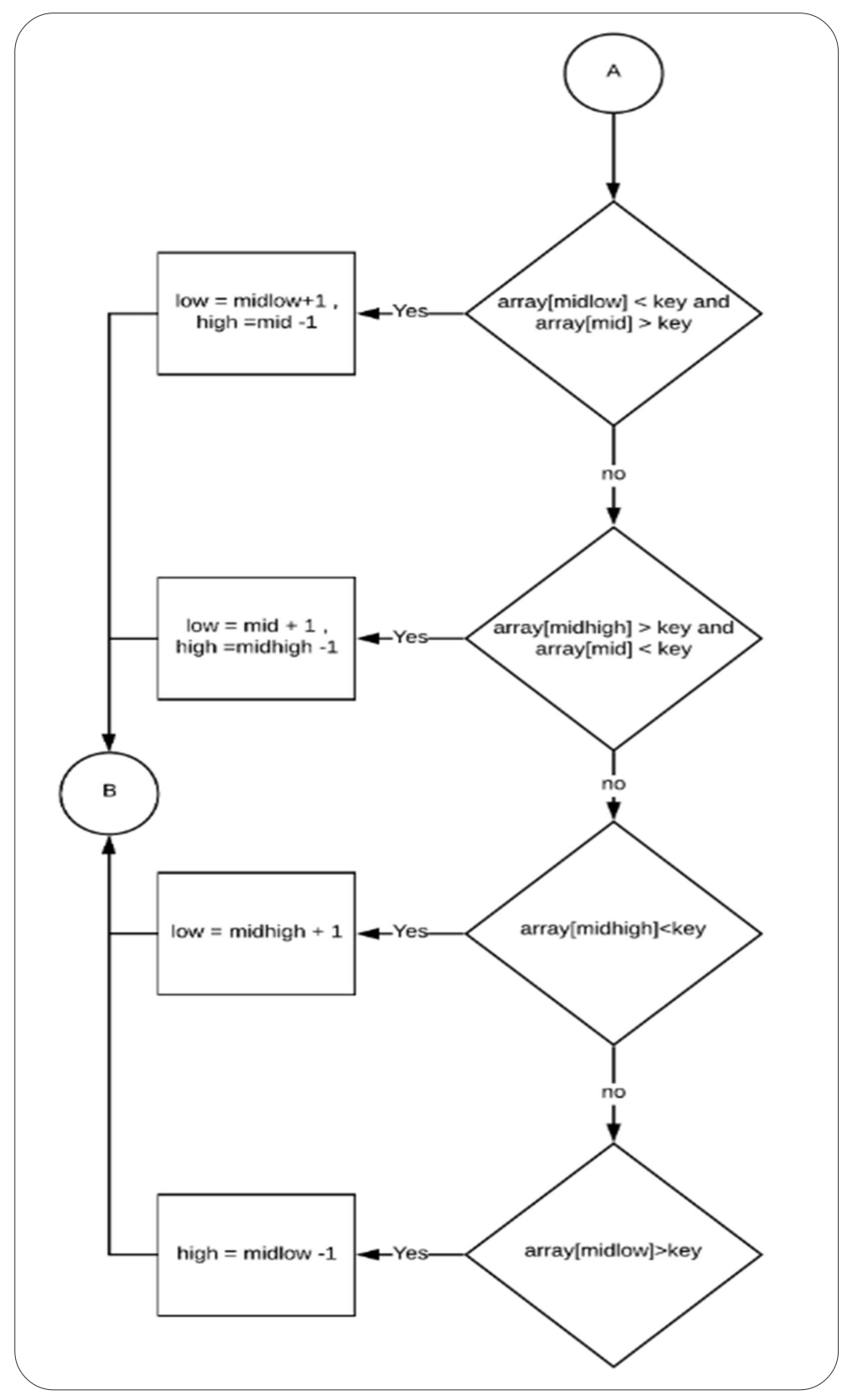

Figure 2. Second part flowchart of modified binary search

- Unlike the binary search algorithm, that would otherwise have come across the data sought if the data sought is located in the mid variable, (Balogun, 2013) the modified binary search algorithm will have the data found when the sought data is in the position of mid-low, mid, and mid-high variables.

-When the sought data is in between low and mid-low variable, then the variable is equal to mid-low high-1

-When the sought data is between mid-low and mid variables, the low variable will be equal to mid-low +1 , and the high is equal to the mid-1

-When the sought data is between mid and mid-high variable, then the variable low will be equal to mid +1 , and the high is equal to mid-high-1.
-When the sought is between the mid-high and high variables, then the low variable will be equal to mid- high +1 .

And it can be seen an illustration of how the modified binary search works. In the following illustration, search for key search 4.

And if there is 15 array data and searching for data 7 .

As can be seen from Figures 1 and 2 that the modified binary search algorithm has more calculations than the original binary search but it can be seen that in Figures 3 and 4 it has a shorter iteration.

\section{Result}

a) The modified binary search algorithm will be compared 
with the original binary search algorithm and other data searching algorithms, such as the Linear Interpolation algorithm. This is done so this research can prove what kind of algorithm is faster in finding data.

b) It is compared by using the performance.now() JavaScript function, which is declared in the beginning and the end of the process to be observed so that the algorithm is easy to calculate the required duration algorithm for solving a problem(Schwarz et al., 2017).

c) This research will test any algorithms that have been determined using the data of 100.000 sorted numerical data values as said in the discussion from the previous chapter and will at first search for the number 25138 in the database or the provided array.

The results from Figure 5 until Figure 8 are shown that some algorithms with a different method will give different times result to search number 25138 in 100.000 sorted numerical data. And the result is shown in Figure 5, with the linear searching algorithm method needs 5.624999990686774 milliseconds for the data to be found, and for Interpolation Search that showed in Figure 6 need 0.1550000160932541 milliseconds. And for binary search algorithm that is shown in Figure 7 need 0.2899999963119626 milliseconds to found the number, and the last algorithm that we discuss in this research, just only need 0.1399999891873449 milliseconds to found the number, it is shown in Figure 8.

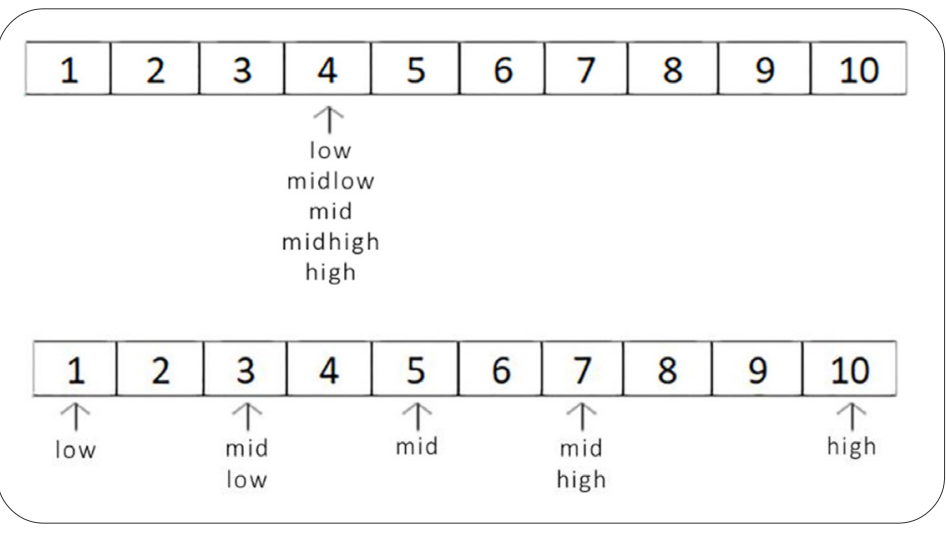

Figure 3. Simulation of Modified Binary Search With 10 Data

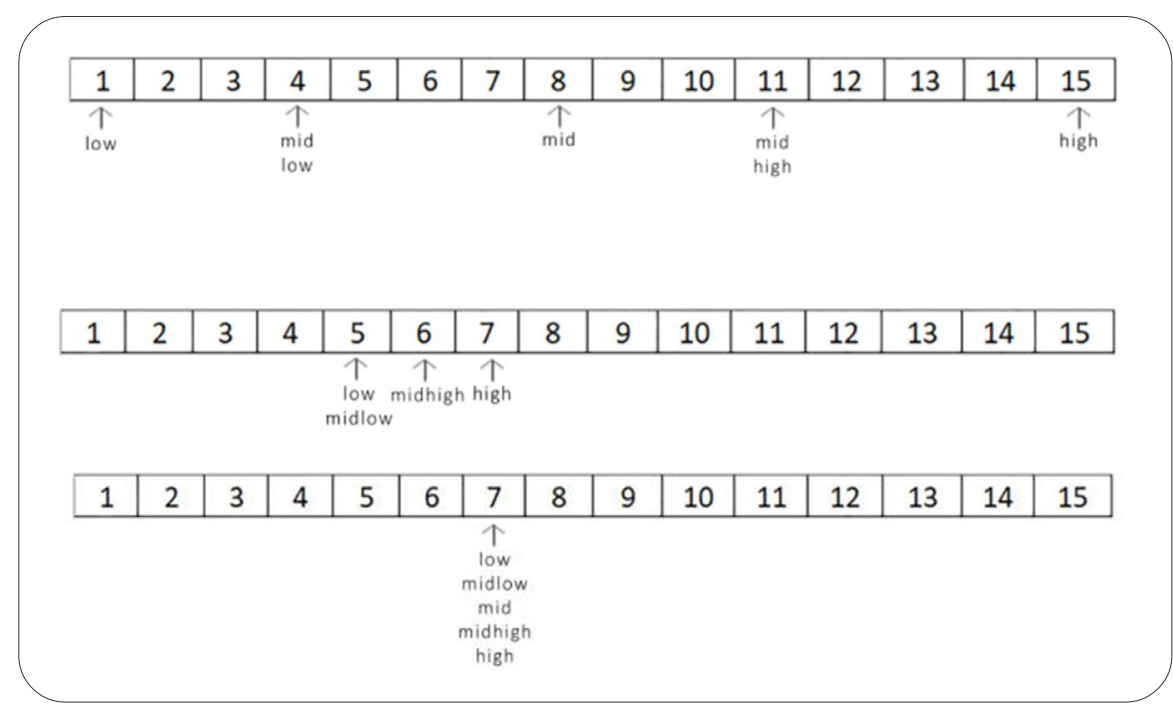

Figure 4. Simulation of Modified Binary Search with 15 Data

Array :

$1,2,3,4,5,6,7,8,9,10,11,12,13,14,15,16,17,18,19,20,21,22,23,24,25,26,27$,

Search : 25138

Found 25138 in index of : 25137

Duration : 5.964999999378051 milliseconds and total iteration : 25139

Figure 5. Linear Search 
Array :

$1,2,3,4,5,6,7,8,9,10,11,12,13,14,15,16,17,18,19,20,21,22,23,24,25,26,27$,

Search : 25138

Found 25138 in index of : 25137

Duration : 0.15500000063184416 milliseconds and total iteration : 2

Figure 6. Interpolation Search

Array :

$1,2,3,4,5,6,7,8,9,10,11,12,13,14,15,16,17,18,19,20,21,22,23,24,25,26,27$,

Search :25138

Found 25138 in index of : 25137

Duration : 0.28500000007625204 milliseconds and total iteration : 17

Figure 7. Binary Search

Array :

$1,2,3,4,5,6,7,8,9,10,11,12,13,14,15,16,17,18,19,20,21,22,23,24,25,26,27$,

Search :25138

Found 25138 in index of : 25137

Duration : 0.13500000022759195 milliseconds and total iteration : 9

Figure 8. Modified Binary Search

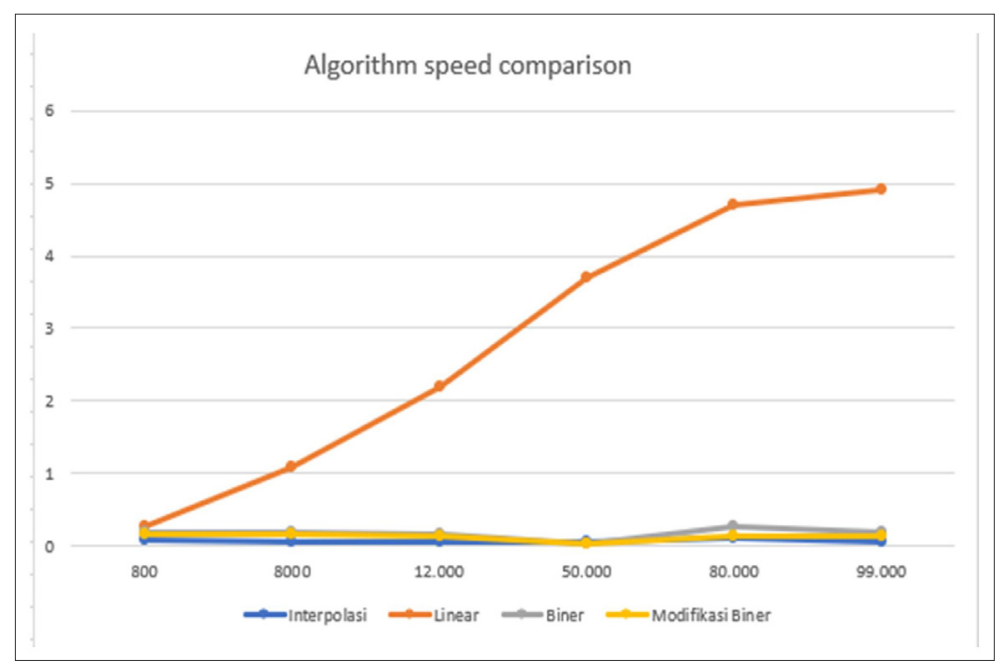

Figure 9. Algorithm speed comparison

It could also be noted that the number of iterations is not too directly proportional to the duration of the searching process of the data, that's because the interpolation search only requires two iterations but not sooner than the modification of the binary search that has nine iterations, it can be analyzed that the possibility of the interpolation search algorithm had a process of repetition which in fewer, but also more complicated.

This study will do another research by replacing the sought data, now the sought data will be $800,8000,12.000$, $50.000,80.000,99.000$. This is done for the sake of getting a more precise result and for consistency from the four searching algorithms that are tested, with the result being displayed here.

Since the chart in Figure 9 that is displayed is not less specific from the comparison of search algorithms from interpolation search, binary search, and the modification of the binary caused due to the distance ratio between the duration of the search algorithm is linear, and others as well as the limitation of the size of the chart, then, it will now only show the chart of interpolation search, binary search algorithms, and the binary search algorithm modification.

From the chart that is shown in Figure 10, it can be concluded that the interpolation search algorithm is more 


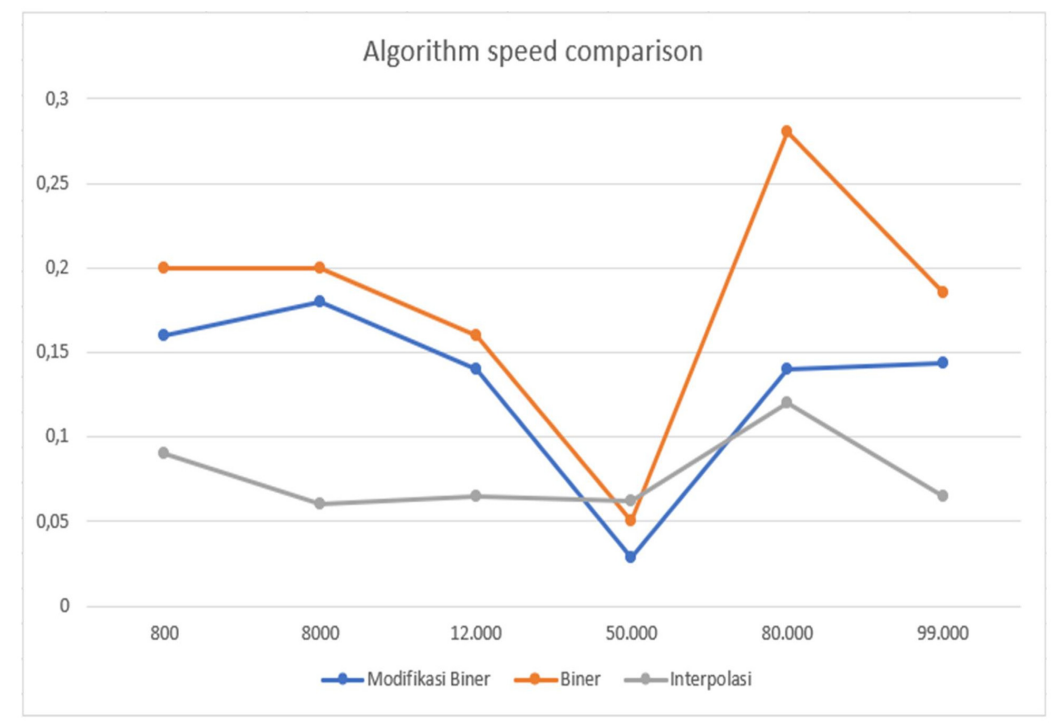

Figure 10. Algorithm speed comparison

consistent and dominantly faster, but there are also some events where the binary search algorithm and the modification of binary search algorithms have much faster performance than the interpolation search algorithm. From the chart above, it has also been proved that the modified binary search algorithm is faster than ever before.

\section{Conclusion}

It was concluded that the linear search is the slowest algorithm and the interpolation search is the fastest algorithm by comparing the four searching algorithms. However, in several cases, linear search algorithm becomes faster, when the data is not sorted, unlike the interpolation search algorithm, which can only be processed with the data that has been sorted from the smallest to the biggest, or from the biggest to smallest and modified binary search has more calculations that are a burden on their algorithms but have far fewer iterations than the original binary search. And it has also been proven that by simply adding the two variables between mid-variables can make a binary search algorithm to be more consistent and faster to solve problems or search the data sector in a database or array.

\section{References}

[1] S. K., B. (2016). Research \& Reviews: Journal of Engineering and Technology Matrix Search Algorithm Using Binary Search Trees. Journal of Engineering and Technology, 5 (2) 3-8.

[2] Balogun, B. G. (2013). Simulating Binary Search Technique Using Different Sorting Algorithms. International Journal of Applied Science and Technology, 3 (6) 67-75.

[3] Lin, A. (2019). Binary search algorithm. Wiki Journal of Science, 2 (1), 5. https://doi.org/10.15347/wjs/2019.005

[4] Marszalek, Z. (2017). Parallelization of modified merge sort algorithm. Symmetry, 9 (9). https://doi.org/10.3390/ sym9090176

[5] Mehlhorn, K., Sanders, P. (2008). Book_Chapter 7: Algorithms and Data Structures. Wikipedians. https:// doi.org/10.1007/978-3-540-77978-0

[6] Mullins, A. P., Gennifer, B., Farrell, E., Baldwin, C. R. (2017). Investigating the Benefits of Parallel Processing for Binary Search. Int'l Conf. Foundations of Computer Science, 38-42.

[7] P. Parmar, V., Kumbharana, C. (2015). Comparing Linear Search and Binary Search Algorithms to Search an Element from a Linear List Implemented through Static Array, Dynamic Array and Linked List. International Journal of Computer Applications, 121 (3) 13-17. https:// doi.org/10.5120/21519-4495

[8] Pooja, P. (2015). A Comparison Based Analysis of Different Types of Sorting and Searching Algorithms in Data Structures. International Journal of Advance Engineering and Research Development, Vol. 2. https://doi.org/ 10.21090/ijaerd.020657

[9] Pushpa, S., Vinod, P. (2007). Binary Search Tree Balancing Methods/: A Critical Study. Journal of Computer Science, 7 (8) 237-243.

[10] Rahim, R., Nurarif, S., Ramadhan, M., Aisyah, S., Purba, W. (2017). Comparison Searching Process of Linear, Binary and Interpolation Algorithm. Journal of Physics: Conference Series, 930 (1). https://doi.org/10.1088/ 1742-6596/930/1/012007

[11] Schwarz, M., Maurice, C., Gruss, D., Mangard, S. (2017). Fantastic timers and where to find them: Highresolution microarchitectural attacks in javascript. Lecture Notes in Computer Science (Including Subseries Lecture Notes in Artificial Intelligence and Lecture Notes in Bioinformatics), 10322 LNCS, 247-267. https://doi.org/ 10.1007/978-3-319-70972-7_13 
[12] Tremblay, J. -P., Sorenson, P. G. (1984). An introduction to data structures with applications. McGraw-Hill Computer Science Series, 861. Retrieved from https:// dl.acm.org/citation.cfm?id=2802
[13] Sandt, Van., P., Chronis, Y., Patel, J. M. (2019). Efficiently searching in-memory sorted arrays: Revenge of the interpolation search? Proceedings of the ACM SIGMOD International Conference on Management of Data, 36-53. https://doi.org/10.1145/3299869.3300075 